\title{
Minimum Cost Homomorphisms to Reflexive Digraphs
}

\author{
Arvind Gupta Pavol Hell Mehdi Karimi Arash Rafiey \\ School of Computing Science \\ Simon Fraser University \\ Burnaby, B.C., Canada, V5A 1S6 *
}

\begin{abstract}
For digraphs $G$ and $H$, a homomorphism of $G$ to $H$ is a mapping $f: V(G) \rightarrow V(H)$ such that $u v \in A(G)$ implies $f(u) f(v) \in A(H)$. If moreover each vertex $u \in V(G)$ is associated with costs $c_{i}(u), i \in V(H)$, then the cost of a homomorphism $f$ is $\sum_{u \in V(G)} c_{f(u)}(u)$. For each fixed digraph $H$, the minimum cost homomorphism problem for $H$, denoted $\operatorname{MinHOM}(H)$, is the following problem. Given an input digraph $G$, together with costs $c_{i}(u), u \in V(G), i \in V(H)$, and an integer $k$, decide if $G$ admits a homomorphism to $H$ of cost not exceeding $k$. Minimum cost homomorphism problems encompass (or are related to) many well studied optimization problems such as chromatic partition optimization and applied problems in repair analysis. For undirected graphs the complexity of the problem, as a function of the parameter $H$, is well understood; for digraphs, the situation appears to be more complex, and only partial results are known. We focus on the minimum cost homomorphism problem for reflexive digraphs $H$ (every vertex of $H$ has a loop). It is known that the problem $\operatorname{MinHOM}(H)$ is polynomial time solvable if the digraph $H$ has a Min-Max ordering, i.e., if its vertices can be linearly ordered by $<$ so that $i<j, s<r$ and $i r, j s \in A(H)$ imply that $i s \in A(H)$ and $j r \in A(H)$. We give a forbidden induced subgraph characterization of reflexive digraphs with a Min-Max ordering; our characterization implies a polynomial time test for the existence of a Min-Max ordering. Using this characterization, we show that for a reflexive digraph $H$ which does not admit a Min-Max ordering, the minimum cost homomorphism problem is NP-complete, as conjectured by Gutin and Kim. Thus we obtain a full dichotomy classification of the complexity of minimum cost homomorphism problems for reflexive digraphs.
\end{abstract}

\section{Introduction and Terminology}

For digraphs $G$ and $H$, a mapping $f: V(G) \rightarrow V(H)$ is a homomorphism of $G$ to $H$ if $u v$ is an arc of $G$ implies $f(u) f(v)$ is an arc of $H$. Let $H$ be a fixed digraph: the homomorphism

*arvind@mitacs.ca , pavol@cs.sfu.ca , mmkarimi@cs.sfu.ca , arashr@cs.sfu.ca 
problem for $H$, denoted $\operatorname{HOM}(H)$, asks whether or not an input digraph $G$ admits a homomorphism to $H$. The list homomorphism problem for $H$, denoted $\operatorname{ListHOM}(H)$, asks whether or not an input digraph $G$, with lists $L_{u} \subseteq V(H), u \in V(G)$, admits a homomorphism $f$ to $H$ in which all $f(u) \in L_{u}, u \in V(G)$.

Suppose $G$ and $H$ are digraphs, and $c_{i}(u), u \in V(G), i \in V(H)$, are real costs. The cost of a homomorphism $f$ of $G$ to $H$ is $\sum_{u \in V(G)} c_{f(u)}(u)$. If $H$ is fixed, the minimum cost homomorphism problem for $H$, denoted $\operatorname{MinHOM}(H)$, is the following problem. Given an input digraph $G$, together with $\operatorname{costs} c_{i}(u), u \in V(G), i \in V(H)$, and an integer $k$, decide if $G$ admits a homomorphism to $H$ of cost not exceeding $k$.

If the graph $H$ is symmetric (each $u v \in A(H)$ implies $v u \in A(H)$ ), we may view $H$ as an undirected graph. In this way, we may view the problem $\operatorname{MinHOM}(H)$ as applying also to undirected graphs.

The minimum cost homomorphism problem was introduced, in the context of undirected graphs, in [16]. There, it was motivated by a real-world problem in defense logistics; in general, the problem seems to offer a natural and practical way to model many optimization problems. Special cases include for instance the list homomorphism problem [19, 21] and the optimum cost chromatic partition problem [18, 24, 25] (which itself has a number of well-studied special cases and applications [27, 29]).

Our interest is in proving dichotomies: given a class of problems such as $\operatorname{HOM}(H)$, we would like to prove that for each digraph $H$ the problem is polynomial-time solvable, or NP-complete. This is, for instance, the case for $\operatorname{HOM}(H)$ with undirected graphs $H$ [20]; in that case it is known that $\operatorname{HOM}(H)$ is polynomial time solvable when $H$ is bipartite or has a loop, and NP-complete otherwise [20]. This is a dichotomy classification, since we specifically classify the complexity of the problems $\operatorname{HOM}(H)$, depending on $H$.

For undirected graphs $H$, a dichotomy classification for the problem $\operatorname{MinHOM}(H)$ has been provided in [17]. (For $\operatorname{ListHOM}(H)$, consult [6].) Thus, the minimum cost homomorphism problem for graphs has been handled, and interest shifted to directed graphs. The first studies [13, 14, 15] focused on irreflexive digraphs (no vertex has a loop), where dichotomies has been obtained for digraphs $H$ such that $U(H)$ is a complete or complete multipartite graph. More recently, [11] promoted the study of digraphs with loops allowed; and, in particular, of reflexive digraphs. Dichotomy has been proved for reflexive digraphs $H$ such that $U(H)$ is a complete graph, or a complete multipartite graph without digons [10, 12]. In this paper, we give a full dichotomy classification of the complexity of $\operatorname{MinHOM}(H)$ for reflexive digraphs; this is the first dichotomy result for a general class of digraphs - our only restriction is that the digraphs are reflexive. The dichotomy classification we prove verifies a conjecture of Gutin and Kim [10]. (Partial results on $\operatorname{ListHOM}(H)$ for digraphs can be found in [3, 5, 17, 8, 9, 23, 32].

Let $H$ be any digraph. An $\operatorname{arc} x y \in A(H)$ is symmetric if $y x \in A(H)$; the digraph $H$ is symmetric if each arc of $H$ is symmetric. Otherwise, we denote by $S(H)$ the symmetric 


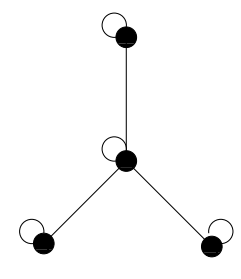

a) Claw

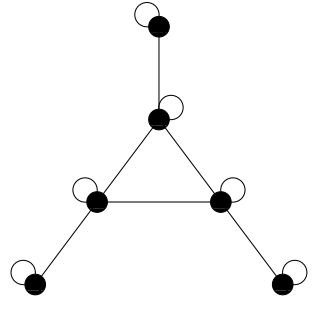

b) Net

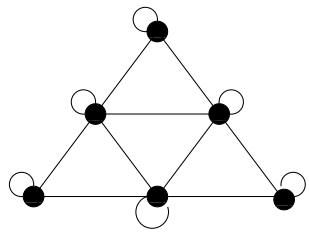

c) Tent

Figure 1: The claw, the net, and the tent.

subgraph of $H$, i.e., the undirected graph with $V(S(H))=V(H)$ and $E(S(H))=\{u v$ : $u v \in A(H)$ and $v u \in A(H)\}$. We also denote by $U(H)$ the underlying graph of $H$, i.e., the undirected graph with $V(U(H))=V(H)$ and $E(U(H))=\{u v: u v \in A(H)$ or $v u \in A(H)\}$. If $H$ is a reflexive digraph, then both $S(H)$ and $U(H)$ are reflexive graphs. Finally, we denote by $B(H)$ the bipartite graph obtained from $H$ as follows. Each vertex $v$ of $H$ gives rise to two vertices of $B(H)$ - a white vertex $v^{\prime}$ and a black vertex $v^{\prime \prime}$; each arc $v w$ of $H$ gives rise to an edge $v^{\prime} w^{\prime \prime}$ of $B(H)$. Note that if $H$ is a reflexive digraph, then all edges $v^{\prime} v^{\prime \prime}$ are present in $B(H)$. The converse of $G$ is the digraph obtained from $G$ by reversing the directions of all arcs.

We say that an undirected graph $H$ is a proper interval graph if there is an inclusionfree family of intervals $I_{v}, v \in V(H)$, such that $v w \in E(H)$ if and only if $I_{v}$ intersects $I_{w}$. Note that by this definition proper interval graphs are reflexive. Wegner proved [30] that a reflexive graph $H$ is a proper interval graph if and only if it does not contain an induced cycle $C_{k}$, with $k \geq 4$, or an induced claw, net, or tent, as given in Figure 1 .

We say that a bipartite graph $H$ (with a fixed bipartition into white and black vertices) is a proper interval bigraph if there are two inclusion-free families of intervals $I_{v}$, for all white vertices $v$, and $J_{w}$ for all black vertices $w$, such that $v w \in E(H)$ if and only if $I_{v}$ intersects $J_{w}$. By this definition proper interval bigraphs are irreflexive and bipartite. A Wegner-like characterization (in terms of forbidden induced subgraphs) of proper interval bigraphs is given in [22]: $H$ is a proper interval bigraph if and only if it does not contain an induced cycle $C_{2 k}$, with $k \geq 3$, or an induced biclaw, binet, or bitent, as given in Figure 2.

A linear ordering $<$ of $V(H)$ is a Min-Max ordering if $i<j, s<r$ and $i r, j s \in A(H)$ imply that $i s \in A(H)$ and $j r \in A(H)$. For a reflexive digraph $H$, it is easy to see that $<$ is a Min-Max ordering if and only if for any $j$ between $i$ and $k$, we have $i k \in A(H)$ imply $i j, j k \in A(H)$. For a bipartite graph $H$ (with a fixed bipartition into white and black vertices), it is easy to see that $<$ is a Min-Max ordering if and only if $<$ restricted to the white vertices, and < restricted to the black vertices satisfy the condition of Min-Max 


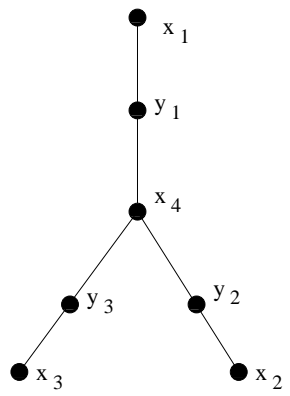

a) Biclaw

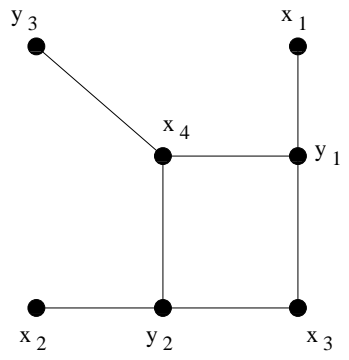

b) Binet

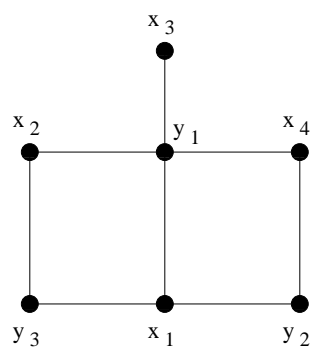

c) Bitent

Figure 2: The biclaw, the binet, and the bitent.

orderings, i.e., $i<j$ for white vertices, and $s<r$ for black vertices, and $i r, j s \in A(H)$, imply that $i s \in A(H)$ and $j r \in A(H)$ ). A bipartite Min-Max ordering is an ordering < specified just for white and for black vertices.

It is known that if $H$ admits a Min-Max ordering, then the problem $\operatorname{MinHOM}(H)$ is polynomial time solvable [13], see also [4, 26]; however, there are digraphs with polynomial $\operatorname{MinHOM}(H)$ which do not have Min-Max ordering [14. For undirected graphs, all $H$ without a Min-Max ordering yield an NP-complete $\operatorname{MinHOM}(H)$ [17]; moreoever, having a Min-Max ordering can be characterized by simple forbidden induced subgraphs, and recognized in polynomial time [17]. In particular, a reflexive graph admits a Min-Max ordering if and only if it is a proper interval graph, and a bipartite graph admits a MinMax ordering if and only if it is a proper interval bigraph [17].

We shall give a combinatorial description of reflexive digraphs with Min-Max ordering, in terms of forbidden induced subgraphs. Our characterization yields a polynomial time algorithm for the existence of a Min-Max ordering in a reflexive digraph. It also allows us to complete a dichotomy classification of $\operatorname{MinHOM}(H)$ for reflexive digraphs $H$, by showing that all problems $\operatorname{MinHOM}(H)$ where $H$ does not admit a Min-Max ordering are NP-complete. This verifies a conjecture of Gutin and Kim in [10].

\section{Structure and Forbidden Subgraphs}

Since both reflexive and bipartite graphs admit a characterization of existence of Min-Max orderings by forbidden induced subgraphs, our goal will be accomplished by proving the following theorem. It also implies a polynomial time algorithm to test if a reflexive digraph has a Min-Max ordering.

Theorem 2.1 A reflexive digraph $H$ has a Min-Max ordering if and only if 


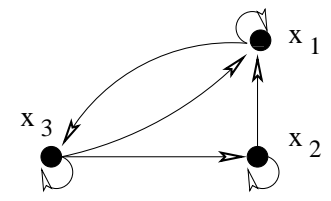

$\mathrm{H}_{1}$

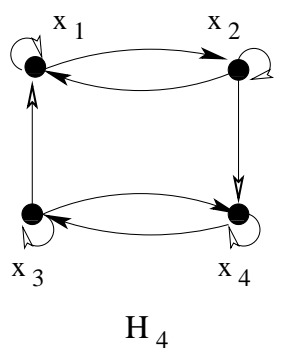

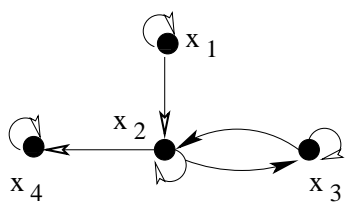

$\mathrm{H}_{2}$

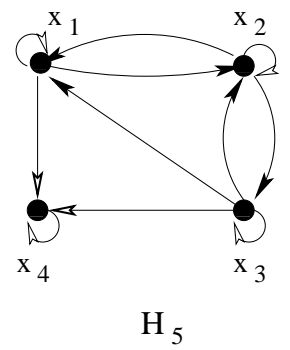

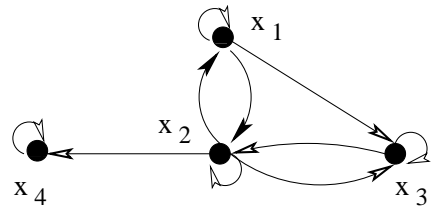

$\mathrm{H}_{3}$

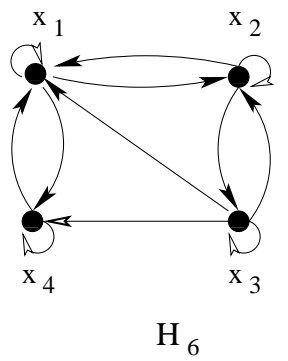

Figure 3: The obstructions $H_{i}$ with $i=1,2,3,4,5,6$.

- $S(H)$ is a proper interval graph, and

- $B(H)$ is a proper interval bigraph, and

- $H$ does not contain an induced subgraph isomorphic to $H_{i}$ with $i=1,2,3,4,5,6$.

The digraphs $H_{i}$ are depicted in Figure 3. The resulting forbidden subgraph characterization is summarized in the following corollary. Note that forbidden subgraphs in $S(H)$ directly describe forbidden subgraphs in $H$, and it is easy to see that each forbidden induced subgraph in $B(H)$ can also be translated to a small family of forbidden induced subgraphs in $H$.

Corollary 2.2 A reflexive digraph $H$ has a Min-Max ordering if and only if $S(H)$ does not contain an induced $C_{k}, k \geq 4$, or claw, net, or tent, $B(H)$ does not contain an induced $C_{2 k}, k \geq 3$, or biclaw, binet, or bitent, and $H$ does not contain an induced $H_{i}$ with $i=$ $1,2,3,4,5,6$.

We proceed to prove the Theorem.

Proof: Suppose first that $<$ is a Min-Max ordering $<$ of $H$. It is easily seen that $<$ is also a Min-Max ordering of $S(H)$, and that < applied separately to the corresponding white and black vertices of $B(H)$ is a bipartite Min-Max ordering of $B(H)$. To complete the proof of necessity, we now claim that none of the digraphs $H_{i}, i=1,2,3,4,5,6$ admits a Min-Max ordering. We only show this for $\mathrm{H}_{3}$, the proofs of the other cases being similar. 
Suppose that $<$ is a Min-Max ordering of $H_{3}$. For the triple $x_{1}, x_{2}, x_{3}$, we note that $x_{2}$ must be between $x_{1}$ and $x_{3}$ in the ordering $<$, as otherwise we would have $x_{1} x_{3} \in E(S(H))$ . Without loss of generality assume that $x_{1}<x_{2}<x_{3}$. Since $x_{1}$ and $x_{4}$ are independent and $x_{1} x_{2} \in E(S(H))$, we must have $x_{4}>x_{1}$. A similar argument yields $x_{4}<x_{3}$; however, $x_{1}<x_{4}<x_{3}$ is impossible, as $x_{1} x_{3} \in A(H)$ but $x_{1} x_{4} \notin A(H)$.

To prove the sufficiency of the three conditions, we shall prove the following claim.

Lemma 2.3 If $S(H)$ has a Min-Max ordering and $B(H)$ has a bipartite Min-Max ordering, then either $H$ has a Min-Max ordering, or $H$ contains an induced $H_{i}$ (or its converse) for some $i=1,2,3,4,5,6$.

Proof: Suppose < is a bipartite Min-Max ordering of $B(H)$. A pair $u, v$ of vertices of $H$ is proper for $<$ if $u^{\prime}<v^{\prime}$ if and only if $u^{\prime \prime}<v^{\prime \prime}$ in $B(H)$. We say a bipartite Min-Max ordering $<$ is proper if all pairs $u, v$ of $H$ are proper for $<$. If $<$ is a proper bipartite Min-Max ordering, then we can define a corresponding ordering $\prec$ on the vertices of $H$, where $u \prec v$ if and only if $u^{\prime}<v^{\prime}$ (which happens if and only if $u^{\prime \prime}<v^{\prime \prime}$ ). It is easy to check that $\prec$ is now a Min-Max ordering of $H$.

Suppose, on the other hand, that the bipartite Min-Max ordering $<$ on $B(H)$ is not proper. Thus there are vertices $v^{\prime}, u^{\prime}$ such that $v^{\prime}<u^{\prime}$ and $u^{\prime \prime}<v^{\prime \prime}$. Suppose there is no vertex $s^{\prime}$ such that $s^{\prime} v^{\prime \prime} \in E(B(H)), s^{\prime} u^{\prime \prime} \notin E(B(H))$ : then we can exchange the position of $v^{\prime \prime}$ and $u^{\prime \prime}$ in $<$ and still have a bipartite Min-Max ordering. Furthermore, this exchange strictly increases the number of proper pairs in $H$ : any $w$ with $u^{\prime \prime}<w^{\prime \prime}<v^{\prime \prime}$ and $u^{\prime}<w^{\prime}$ creates a new improper pair $u, w$ but also creates a new proper pair $v, w$ (and the pair $u, v$ is also a new proper pair). Analogously, if there is no vertex $t^{\prime \prime}$ such that $u^{\prime} t^{\prime \prime} \in E(B(H))$, $v^{\prime} t^{\prime \prime} \notin E(B(H))$, we can exchange $u^{\prime}, v^{\prime}$ and increase the number of proper pairs in $H$. Suppose we have performed all exchanges until we reached a bipartite Min-Max ordering $<$ which admits no more exchanges. Then there are two possibilities: either $<$ is now proper, and $H$ admits a Min-Max ordering as above, or $<$ is still not proper, and one of the following two cases must occur (up to symmetry):

Case 1: $s^{\prime} v^{\prime \prime}, v^{\prime} t^{\prime \prime} \in E(B(H))$ and $s^{\prime} u^{\prime \prime}, u^{\prime} t^{\prime \prime} \notin E(B(H))$.

It is easy to see that since $<$ is a bipartite Min-Max ordering, we must have $u^{\prime}<s^{\prime}$ and $t^{\prime \prime}<u^{\prime \prime}$. (Note that means that $s^{\prime \prime} \neq t^{\prime \prime}$.) Since $u^{\prime} u^{\prime \prime}, v^{\prime} v^{\prime \prime} \in E(B(H)$ ), by the same argument we must have $u^{\prime} v^{\prime \prime}, v^{\prime} u^{\prime \prime} \in E(B(H))$; and similarly we obtain $s^{\prime} t^{\prime \prime} \notin E(B(H))$. If both $v^{\prime} s^{\prime \prime}$ and $t^{\prime} v^{\prime \prime}$ are edges of $B(H)$ then $u, v, s, t$ induce a claw in $S(H)$ : indeed in $B(H)$, we have the edges $v^{\prime} t^{\prime \prime}, t^{\prime} v^{\prime \prime}, v^{\prime} u^{\prime \prime}, u^{\prime} v^{\prime \prime}, v^{\prime} s^{\prime \prime}, s^{\prime} v^{\prime \prime}$ and the non-edges $u^{\prime} t^{\prime \prime}, s^{\prime} u^{\prime \prime}, s^{\prime} t^{\prime \prime}$. This is a contradiction, as $S(H)$ is assumed to have a Min-Max ordering, i.e., be a proper interval graph.

If neither $v^{\prime} s^{\prime \prime}$ nor $t^{\prime} v^{\prime \prime}$ is an edge of $B(H)$, then if $u^{\prime} s^{\prime \prime}$ is an edge of $B(H)$, then $s, v, u$ induce a copy of $H_{1}$ in $H$, and if,$t^{\prime} u^{\prime \prime}$ is an edge of $B(H)$, then $t, v, u$ induce a 
copy of $H_{1}$. Thus consider the case when $u^{\prime} s^{\prime \prime}, t^{\prime} u^{\prime \prime} \notin E(B(H))$. If $t^{\prime} s^{\prime \prime} \in E(B(H))$, then $s^{\prime}, s^{\prime \prime}, t^{\prime}, t^{\prime \prime}, v^{\prime}, v^{\prime \prime}$ would induce a copy of $C_{6}$ in $B(H)$, contrary to our assumption that $B(H)$ has a bipartite Min-Max ordering, i.e., is a proper interval bigraph. Thus $t^{\prime} s^{\prime \prime} \notin E(B(H))$ and $t, s, v, u$ induce a copy of $H_{2}$ in $H$.

If only one of $v^{\prime} s^{\prime \prime}$ or $t^{\prime} v^{\prime \prime}$ is an edge of $B(H)$, assume first that $v^{\prime} s^{\prime \prime} \in E(B(H))$ and $t^{\prime} v^{\prime \prime} \notin E(B(H))$. If $t^{\prime} u^{\prime \prime}$ is an edge of $B(H)$, then $t, v, u$ induce a copy of $H_{1}$ in $H$, and if $t^{\prime} s^{\prime \prime}$ is an edge of $B(H)$, then $t, v, s$ similarly induce a copy of $H_{1}$; thus asume that $t^{\prime} u^{\prime \prime}, t^{\prime} s^{\prime \prime} \notin E(B(H))$. Note that $u^{\prime} s^{\prime \prime} \in E(B(H))$, else the vertices $u^{\prime}, u^{\prime \prime}, v^{\prime}, t^{\prime \prime}, t^{\prime}, s^{\prime \prime}, s^{\prime}$ would induce a biclaw in $B(H)$, contrary to $B(H)$ being a proper interval bigraph. It now follows that $s, t, u, v$ induce a copy of $H_{3}$ in $H$. If $v^{\prime} s^{\prime \prime} \notin E(B(H))$ and $t^{\prime} v^{\prime \prime} \in E(B(H))$, the proof is similar, except we obtain copies of $H_{1}$ and the converse of $H_{3}$.

Case 2: $s^{\prime} v^{\prime \prime}, u^{\prime} t^{\prime \prime} \in E(B(H))$ and $s^{\prime} u^{\prime \prime}, v^{\prime} t^{\prime \prime} \notin E(B(H))$.

We again easily observe that we must have $u^{\prime}<s^{\prime \prime}, v^{\prime \prime}<t^{\prime \prime}$, and $u^{\prime} v^{\prime \prime}, v^{\prime} u^{\prime \prime} \in E(B(H))$. If $s^{\prime \prime}=t^{\prime \prime}$ we obtain a copy of $H_{1}$ induced by $u, v, s$ in $H$; hence we assume that $s^{\prime \prime} \neq$ $t^{\prime \prime}$. Suppose first that $u^{\prime} s^{\prime \prime}, t^{\prime} v^{\prime \prime} \notin E(B(H))$. We have $s^{\prime}<t^{\prime}$ and $t^{\prime \prime}<s^{\prime \prime}$, and so $t^{\prime} s^{\prime \prime}, s^{\prime} t^{\prime \prime} \in A(H)$, implying that $u, v, s, t$ induce a copy of $H_{4}$ in $H$. Suppose next that both $t^{\prime} v^{\prime \prime}, u^{\prime} s^{\prime \prime} \in E(B(H))$. If $v^{\prime} s^{\prime \prime}$ is not an edge of $B(H)$, vertices $u, v, s$ induce a copy of $H_{1}$ in $H$, and if $t^{\prime} u^{\prime \prime}$ is not an edge of $B(H)$, vertices $u, v, t$ induce a copy of $H_{1}$ in $H$. Thus we have $v^{\prime} s^{\prime \prime}, t^{\prime} u^{\prime \prime} \in E(B(H))$. Now we have $t^{\prime}<s^{\prime}$ and $s^{\prime \prime}<t^{\prime \prime}$, and hence $t^{\prime} s^{\prime \prime}, s^{\prime} t^{\prime \prime} \in E(B(H))$. This is impossible, since $u, v, s, t$ would induce a copy of $C_{4}$ in $S(H)$. Finally, if only one of $t^{\prime} v^{\prime \prime}, u^{\prime} s^{\prime \prime}$ as an edge of $B(H)$, say $u^{\prime} s^{\prime \prime} \in E(B(H))$ and $t^{\prime} v^{\prime \prime} \notin E(B(H))$ (the other case is symmetric), then with the same argument as above, $v^{\prime} s^{\prime \prime} \in E(B(H)), s^{\prime} t^{\prime \prime} \in E(B(H))$, and $s, t, u, v$ induce (depending on which of the pairs $t^{\prime} u^{\prime \prime}, t^{\prime} s^{\prime \prime}$ are edges of $\left.B(H)\right)$ one of $H_{1}, H_{5}$ (or its converse), or $H_{6}$ (or its converse). $\diamond$

\section{Complexity}

If $H$ has a Min-Max ordering, then $\operatorname{MinHOM}(H)$ is polynomial time solvable [13 see also [4, 26]. Now using our forbidden induced subgraph characterization we can prove that reflexive digraphs $H$ without a Min-Max ordering yield NP-complete $\operatorname{MinHOM}(H)$ problems. Note that we already know that $\operatorname{MinHOM}(S(H))$ is NP-complete if $S(H)$ is not a proper interval graph, and $\operatorname{MinHOM}(B(H))$ is NP-complete if $B(H)$ is not a proper interval bigraph 13 . We begin with a few simple observations. They first one is easily proved by setting up a natural polynomial time reduction from $\operatorname{MinHOM}(B(H))$ to $\operatorname{MinHOM}(H)[11$.

Proposition 3.1 [11] If $\operatorname{MinHOM}(B(H))$ is $N P$-complete, then $\operatorname{MinHOM}(H)$ is also NP-complete. 
The next two observations are folklore, and proved by obvious reductions, cf. [10].

Proposition 3.2 If $\operatorname{MinHOM}(S(H))$ is $N P$-complete, then $\operatorname{MinHOM}(H)$ is also NPcomplete.

Proposition 3.3 Let $H^{\prime}$ be an induced subgraph of the digraph $H$. If $\operatorname{MinHOM}\left(H^{\prime}\right)$ is NP-complete then $\operatorname{MinHOM}(H)$ is NP-complete.

We now continue to prove that $\operatorname{MinHOM}(H)$ is NP-complete for digraphs $H=H_{1}, \ldots, H_{6}$. Let $\mathcal{I}$ denote the following decision problem: given a graph $X$ and an integer $k$, decide whether or not $X$ contains an independent set of $k$ vertices. This problem has been useful for proving NP-completeness of minimum cost homomorphism problems for undirected graphs [17], and we use it again for digraphs.

Proposition 3.4 [17] The problem $\mathcal{I}$ is NP-complete, even when restricted to threecolourable graphs (with a given three-colouring).

We denote by $\mathcal{I}_{3}$ the restriction of $\mathcal{I}$ to graphs with a given three-colouring. In the following Lemmas, we give a polynomial time reductions from $\mathcal{I}_{3}$. Note that all problems $\operatorname{MinHOM}(H)$ are in NP. The NP-completeness of $\operatorname{MinHOM}\left(H_{1}\right)$ follows from [10], Lemma $2-4$.

Lemma 3.5 The problem MinHOM(H $\left.H_{2}\right)$ is NP-complete.

Proof: We now construct a polynomial time reduction from $\mathcal{I}_{3}$ to $\operatorname{MinHOM}\left(H_{2}\right)$. Let $X$ be a graph whose vertices are partitioned into independent sets $U, V, W$, and let $k$ be a given integer. We construct an instance of $\operatorname{MinHOM}\left(H_{2}\right)$ as follows: the digraph $G$ is obtained from $X$ by replacing each edge $u v$ of $X$ with $u \in U, v \in V$ by an arc $u v$, replacing each edge $u w$ of $X$ with $u \in U, w \in W$ by an arc $u w$, and replacing each edge $v w$ of $X$ with $v \in V, w \in W$ by an arc $w v$. The costs are defined by (writing for simplicity $c_{i}(y)$ for $\left.c_{x_{i}}(y)\right) c_{1}(u)=0, c_{2}(u)=1$ for $u \in U, c_{4}(v)=0, c_{2}(v)=1$ for $v \in V$, and $c_{3}(w)=0, c_{2}(w)=1$, for $w \in W$. All other $c_{i}(y)=|V(X)|$.

We now claim that $X$ has an independent set of size $k$ if and only if $G$ admits a homomorphism to $H_{2}$ of cost $|V(X)|-k$. Let $I$ be an independent set in $G$. We can define a mapping $f: V(G) \rightarrow V\left(H_{2}\right)$ as follows:

- $f(u)=x_{1}$ for $u \in U \cap I$ and $f(u)=x_{2}$ for $u \in U-I$

- $f(v)=x_{4}$ for $v \in V \cap I$ and $f(v)=x_{2}$ for $v \in V-I$ 
- $f(w)=x_{3}$ for $w \in W \cap I$ and $f(w)=x_{2}$ for $w \in W-I$

This is a homomorphism of $G$ to $H_{2}$ of cost $|V(X)|-k$.

Let $f$ be a homomorphism of $G$ to $H_{2}$ of cost $|V(X)|-k$. If $k \leq 0$ then we are trivially done so assume that $k>0$, which implies that all individual costs are either zero or one. Let $I=\left\{y \in V(X) \mid c_{f(y)}(y)=0\right\}$ and note that $|I| \geq k$. It can be seen that $I$ is an independent set in $G$, as if $u v \in E(G)$, where $u \in I \cap U$ and $v \in I \cap V$ then $f(u)=x_{1}$ and $f(v)=x_{4}$, contrary to $f$ being a homomorphism.

Lemma 3.6 MinHOM(H3) is NP-complete.

Proof: The reduction from the proof of Lemma 3.5 also applies here.

Lemma 3.7 MinHOM(H $\left.H_{4}\right)$ is NP-complete.

Proof: We now construct a polynomial time reduction from $\mathcal{I}_{3}$ to $\operatorname{MinHOM}\left(H_{4}\right)$. Let $X$ be a graph whose vertices are partitioned into independent sets $U, V, W$, and let $k$ be a given integer. An instance of $\operatorname{MinHOM}\left(H_{4}\right)$ is formed as follows: the digraph $G$ is obtained from $X$ by replacing each edge $u v$ of $X$ with $u \in U, v \in V$ by an arc $v u$, replacing each edge $u w$ of $X$ with $u \in U, w \in W$ by a directed path $u m_{u w} w$, and replacing each edge $v w$ of $X$ with $v \in V, w \in W$ by a directed path $v m_{v w} w$. The costs are defined by $c_{1}(u)=1, c_{3}(u)=0$ for $u \in U ; c_{2}(v)=0, c_{3}(v)=1$ for $v \in V ; c_{4}(w)=0, c_{1}(w)=1$ for $w \in W ; c_{3}\left(m_{u w}\right)=c_{4}\left(m_{u w}\right)=|V(X)|$ for each edge $u w$ of $X$ with $u \in U, w \in W$; $c_{2}\left(m_{v w}\right)=c_{4}\left(m_{v w}\right)=|V(X)|$ for each edge $v w$ of $X$ with $v \in V, w \in W$; and $c_{i}(m)=0$ for any other vertex $m \in V(G)-V(X)$, and $c_{i}(y)=|V(X)|$ for any other vertex $y \in V(X)$.

We now claim that $X$ has an independent set of size $k$ if and only if $G$ admits a homomorphism to $H_{4}$ of cost $|V(X)|-k$. Let $I$ be an independent set in $G$. We can define a mapping $f: V(G) \rightarrow V\left(H_{2}\right)$ as follows:

- $f(u)=x_{3}$ for $u \in U \cap I$ and $f(u)=x_{1}$ for $u \in U-I$

- $f(v)=x_{2}$ for $v \in V \cap I$ and $f(v)=x_{3}$ for $v \in V-I$

- $f(w)=x_{4}$ for $w \in W \cap I$ and $f(w)=x_{1}$ for $w \in W-I$

- $f\left(m_{u w}\right)=x_{2}$ when $f(u)=x_{1}$, and $f\left(m_{u w}\right)=x_{1}$ when $f(u)=x_{3}$ for each edge $u w$ of $X$ with $u \in U, w \in W$

- $f\left(m_{v w}\right)=x_{3}$ when $f(w)=x_{4}$ and $f\left(m_{v w}\right)=x_{1}$ when $f(w)=x_{1}$ for each edge $v w$ of $X$ with $v \in V, w \in W$ 
This is a homomorphism of $G$ to $H_{4}$ of cost $|V(X)|-k$.

Let $f$ be a homomorphism of $G$ to $H_{4}$ of cost $|V(X)|-k$. We may again assume that all individual costs are either zero or one. Let $I=\left\{y \in V(X) \mid c_{f(y)}(y)=0\right\}$ and note that $|I| \geq k$. It can be again seen that $I$ is an independent set in $G$, as if $u w \in E(G)$, where $u \in I \cap U$ and $w \in I \cap V$ then $f(u)=x_{3}$ and $f(w)=x_{4}$, thus, $f\left(m_{u w}\right)=x_{3}$ or $f\left(m_{u w}\right)=x_{4}$. However, the cost of homomorphism is greater than $|V(X)|$, a contradiction. The other cases can also be treated similarly.

Lemma 3.8 MinHOM(H5) is NP-complete.

Proof: We similarly construct a polynomial time reduction from $\mathcal{I}_{3}$ to $\operatorname{MinHOM}\left(H_{5}\right)$ : this time the digraph $G$ is obtained from $X$ by replacing each edge $u v$ of $X$ with $u \in U, v \in$ $V$ by an arc $u v$; replacing each edge $u w$ of $X$ with $u \in U, w \in W$ by $\operatorname{arcs} u m_{u w}, w m_{u w}$; and replacing each edge $w v$ of $X$ with $w \in W, v \in V$ by a directed path $w m_{w v} v$. The costs are $c_{1}(u)=1, c_{2}(u)=0$ for $u \in U ; c_{2}(v)=1, c_{4}(v)=0$ for $v \in V ; c_{3}(w)=1, c_{1}(w)=0$ for $w \in W ; c_{1}\left(m_{u w}\right)=c_{2}\left(m_{u w}\right)=|V(X)|$ for each edge $u w$ of $X$ with $u \in U, w \in W$; $c_{1}\left(m_{w v}\right)=c_{4}\left(m_{w v}\right)=|V(X)|$ for each edge $w v$ of $X$ with $w \in W, v \in V ; c_{i}(m)=0$ for any other vertex $m \in V(G)-V(X)$, and $c_{i}(y)=|V(X)|$ for any other vertex $y \in V(X)$.

We again claim that $X$ has an independent set of size $k$ if and only if $G$ admits a homomorphism to $H_{5}$ of cost $|V(X)|-k$. Let $I$ be an independent set in $G$. We can define a mapping $f: V(G) \rightarrow V\left(H_{2}\right)$ by $f(u)=x_{2}$ for $u \in U \cap I$ and $f(u)=x_{1}$ for $u \in U-I ; f(v)=x_{4}$ for $v \in V \cap I$ and $f(v)=x_{2}$ for $v \in V-I ; f(w)=x_{1}$ for $w \in W \cap I$ and $f(w)=x_{3}$ for $w \in W-I ; f\left(m_{u w}\right)=x_{3}$ when $f(u)=x_{2}$, and $f\left(m_{u w}\right)=x_{4}$ when $f(u)=x_{1}$, for each edge $u w$ of $X$ with $u \in U, w \in W ; f\left(m_{w v}\right)=x_{3}$ when $f(w)=x_{3}$ and $f\left(m_{w v}\right)=x_{2}$ when $f(w)=x_{1}$, for each edge $w v$ of $X$ with $w \in W, v \in V$. This is a homomorphism of $G$ to $H_{5}$ of cost $|V(X)|-k$.

Let $f$ be a homomorphism of $G$ to $H_{5}$ of cost $|V(X)|-k$. Assuming again that all individual costs are either zero or one, let $I=\left\{y \in V(X) \mid c_{f(y)}(y)=0\right\}$ and note that $|I| \geq k$. It can be seen that $I$ is an independent set in $G$, as if $u w \in E(G)$, where $u \in I \cap U$ and $w \in I \cap V$ then $f(u)=x_{2}$ and $f(w)=x_{1}$, thus, $f\left(m_{u w}\right)=x_{1}$ or $f\left(m_{u w}\right)=x_{2}$. However, the cost of homomorphism is greater than $|V(X)|$, a contradiction. The other cases can also be treated similarly.

Lemma 3.9 MinHOM(H6) is NP-complete.

Proof: The proof is again similar, letting the digraph $G$ be obtained from $X$ by replacing each edge $u v$ of $X$ with $u \in U, v \in V$ by an arc $u v$; replacing each edge $u w$ of $X$ with $u \in U, w \in W$ by a directed path $u m_{u w} w$; and replacing each edge $v w$ of $X$ with $v \in V, w \in W$ by an arc $w v$. The costs are defined by $c_{1}(u)=0, c_{2}(u)=1$ for $u \in U$; $c_{3}(v)=0, c_{1}(v)=1$ for $v \in V ; c_{4}(w)=0, c_{3}(w)=1 ; c_{1}\left(m_{u w}\right)=c_{4}\left(m_{u w}\right)=|V(X)|$ 
for each edge $u w$ of $X$ with $u \in U, w \in W$; and letting $c_{i}(m)=0$ for any other vertex $m \in V(G)-V(X)$, and $c_{i}(y)=|V(X)|$ for any other vertex $y \in V(X)$.

It can again be seen that $X$ has an independent set of size $k$ if and only if $G$ admits a homomorphism to $H_{6}$ of cost $|V(X)|-k$ : lettin $I$ be an independent set in $G$, we define a mapping $f: V(G) \rightarrow V\left(H_{2}\right)$ by $f(u)=x_{1}$ for $u \in U \cap I$ and $f(u)=x_{2}$ for $u \in U-I$; $f(v)=x_{3}$ for $v \in V \cap I$ and $f(v)=x_{1}$ for $v \in V-I ; f(w)=x_{4}$ for $w \in W \cap I$ and $f(w)=x_{3}$ for $w \in W-I ; f\left(m_{u w}\right)=x_{3}$ when $f(u)=x_{2}$ and $f\left(m_{u w}\right)=x_{2}$ when $f(u)=x_{1}$ for each edge $u w, u \in U, w \in W$. This is a homomorphism of $G$ to $H_{6}$ of cost $|V(X)|-k$.

Let $f$ be a homomorphism of $G$ to $H_{6}$ of cost $|V(X)|-k$ and assume again that all individual costs are either zero or one. Let $I=\left\{y \in V(X) \mid c_{f(y)}(y)=0\right\}$ and note that $|I| \geq k$. It can again be seen that $I$ is an independent set in $G$.

We have proved the following result, conjectured in [10].

Theorem 3.10 Let $H$ be a reflexive digraph. If $H$ has a Min-Max ordering, then MinHOM $(H)$ is polynomial time solvable; otherwise, it is NP-complete.

\section{References}

[1] V.E. Alekseev and V.V. Lozin, Independent sets of maximum weight in $(p, q)$-colorable graphs, Discrete Mathematics 265 (2003), 351-356.

[2] J. Bang-Jensen and G. Gutin, Digraphs: Theory, Algorithms and Applications, SpringerVerlag, London, 2000.

[3] R. C. Brewster, P. Hell, Homomorphisms to powers of digraphs. Discrete Mathematics. 244 (2002), 31-41.

[4] D. Cohen, M. Cooper, P. Jeavons, and A. Krokhin, A maximal tractable class of soft constraints. J. Artif. Intell. Res. 22 (2004), 1-22.

[5] T. Feder, Homomorphisms to oriented cycles and k-partite satisfiability. SIAM J. Discrete Math 14 (2001) 471-480.

[6] T. Feder, P. Hell, and J. Huang, Bi-arc graphs and the complexity of list homomorphisms, $J$. Graph Theory 42 (2003) $61-80$.

[7] T. Feder, P. Hell and K. Tucker-Nally, Digraph matrix partitions and trigraph homomorphisms. Discrete Applied Mathematics. 154 (2006), 2458-2469.

[8] T. Feder, P. Hell, J. Huang, List homomorphisms to reflexive digraphs, manuscript 2005.

[9] T. Feder, Classification of Homomorphisms to Oriented Cycles and $k$-Partite Satisfiability. SIAM Journal on Discrete Mathematics. 14 (2001), 471-480.

[10] G. Gutin and E.J. Kim, Complexity of the minimum cost homomorphism problem for semicomplete digraphs with possible loops, submitted. 
[11] G. Gutin and E.J. Kim, Introduction to the minimum cost homomorphism problem for directed and undirected graphs, to appear in Lecture Notes of the Ramanujan Math. Society.

[12] G. Gutin and E.J. Kim, On the complexity of the minimum cost homomorphism problem for reflexive multipartite tournaments, submitted.

[13] G. Gutin, A. Rafiey and A. Yeo, Minimum Cost and List Homomorphisms to Semicomplete Digraphs. Discrete Appl. Math. 154 (2006), 890-897.

[14] G. Gutin, A. Rafiey and A. Yeo, Minimum Cost Homomorphisms to Semicomplete Multipartite Digraphs. Submitted to Discrete Applied Math.

[15] G. Gutin, A. Rafiey and A. Yeo, Minimum Cost Homomorphisms to Semicomplete Bipartite Digraphs. Submitted.

[16] G. Gutin, A. Rafiey, A. Yeo and M. Tso, Level of repair analysis and minimum cost homomorphisms of graphs. Discrete Appl. Math. 154 (2006), 881-889.

[17] G. Gutin, P. Hell, A. Rafiey and A. Yeo, A dichotomy for minimum cost graph homomorphisms, to appear in European J. Combin.

[18] M. M. Halldorsson, G. Kortsarz, and H. Shachnai, Minimizing average completion of dedicated tasks and interval graphs. Approximation, Randomization, and Combinatorial Optimization (Berkeley, Calif, 2001), Lecture Notes in Computer Science, vol. 2129, Springer, Berlin, 2001, pp. 114-126.

[19] P. Hell, Algorithmic aspects of graph homomorphisms, in 'Survey in Combinatorics 2003', London Math. Soc. Lecture Note Series 307, Cambridge University Press, 2003, 239 - 276.

[20] P. Hell and J. Nešetřil, On the complexity of $H$-colouring. J. Combin. Theory B 48 (1990), $92-110$.

[21] P. Hell and J. Nešetřil, Graphs and Homomorphisms. Oxford University Press, Oxford, 2004.

[22] P. Hell and J. Huang, Interval bigraphs and circular arc graphs. J. Graph Theory 46 (2004), 313-327.

[23] P. Hell, J. Nešetřil, X. Zhu, Complexity of Tree Homomorphisms. Discrete Applied Mathematics. 70 (1996), 23-36.

[24] K. Jansen, Approximation results for the optimum cost chromatic partition problem. J. Algorithms 34 (2000), 54-89.

[25] T. Jiang and D. B. West, Coloring of trees with minimum sum of colors. J. Graph Theory 32 (1999), 354-358.

[26] S. Khanna, M. Sudan, L. Trevisan, D. Williamson, The approximability of constraint satisfaction problems, SIAM Journal on Computing 30 (2000) 18631920.

[27] L.G. Kroon, A. Sen, H. Deng, and A. Roy, The optimal cost chromatic partition problem for trees and interval graphs, Graph-Theoretic Concepts in Computer Science (Cadenabbia, 1996), Lecture Notes in Computer Science, vol. 1197, Springer, Berlin, 1997, pp. 279-292.

[28] L. Lovász, Three short proofs in graph theory, J. Combin. Theory, Ser. B 19 (1975), 269-271.

[29] K. Supowit, Finding a maximum planar subset of a set of nets in a channel. IEEE Trans. Computer-Aided Design 6 (1987), 93-94. 
[30] G. Wegner, Eigenschaften der nerven homologische-einfactor familien in $R^{n}$, Ph.D. Thesis, Universität Gottigen, Gottigen, Germany (1967).

[31] D. West, Introduction to Graph Theory, Prentice Hall, Upper Saddle River, 1996.

[32] H. Zhou, Characterization of the homomorphic preimages of certain oriented cycles. SIAM Journal on Discrete Mathematics. 6 (1993), 87-99. 